\title{
AUMENTO DE RESOLUCIÓN DE IMÁGENES TÉRMICAS PROCEDENTES DE UAVS MEDIANTE ALGORITMOS DE PANSHARPENING
}

\author{
UAV THERMAL IMAGING RESOLUTION ENHANCEMENT BY PANSHARPENING ALGORITHMS
}

\author{
Javier Raimundo*, Juan F. Prieto, Serafín López-Cuervo \\ Departamento de Ingeniería Topográfica y Cartográfica, Escuela Técnica Superior de Ingenieros en Topografía, Geodesia y \\ Cartografía, Universidad Politécnica de Madrid, Campus Sur, A-3, Km 7, 28031 Madrid, España. ajraimundo@alumnos.upm.es; \\ juanfprieto@upm.es; s.lopezc@upm.es
}

\begin{abstract}
:
Thermal sensors mounted on Unmanned Aerial Vehicles (UAV) provide images with much lower resolution than other sensors typically mounted simultaneously (visual spectrum sensors). This situation causes an inhomogeneity in the information derived from these datasets. Due to physical limitations in thermal sensor's construction, it can reasonably be assumed that the resolution of thermal sensors will not equal that of other sensors (visible and near-infrared spectrum range) in the short and medium term. Since the 1970s a variety of algorithms have been developed in remote sensing to improve the resolution of low-resolution sensors with information from images with higher resolution. These algorithms, originally designed for satellite platforms, are called pansharpening. Previous researches have been made to translate these technics to thermal imaging. The extent of these previous researches was to analyze only one of the many different pansharpening algorithms. In our work, we have studied over ten algorithms from the two main pansharpening families to determine their possibilities, performance, and results when used in thermal imaging, focused on UAV thermal infrared acquisition. Our methodology simulates a lower resolution thermal image, that once combined with visual spectrum images and processed, can be compared with the original resolution infrared thermal image to establish the quality of the fusion product. This methodology has been applied to a UAV thermal-visual spectrum image acquisition campaign over an industrial building near Toledo (Spain), and the quality of the final products has been quantitatively analyzed. Prior research on thermal imaging pansharpening did not monitor performance in measurable and comparable numerical parameters. Their findings were based merely on visual observation and it was impossible to ensure the quality in further processes and analyses using these enhanced images. In our work, we have calculated quality indices of the enhanced thermal images, determining quality values such as those of the BDSD pansharpening algorithm: $\mathrm{RMSE}=7.400, \mathrm{ERGAS}=1.084$, $\mathrm{SAM}=0.048, \mathrm{PSNR}=31.014, \mathrm{UQI}=0.995$. In conclusion, we have validated the potential of pansharpening algorithms to enhance the resolution of thermal images with the help of higher-resolution visible spectrum RGB images.
\end{abstract}

Key words: thermal imaging, resolution enhancement, pansharpening, super-resolution

\section{Resumen:}

Los sensores térmicos montados sobre vehículos aéreos pilotados remotamente proveen imágenes con mucha menos resolución que otros sensores montados usualmente a la vez (sensores en el espectro visible). Esta situación provoca una disparidad en la información derivada de estos conjuntos de datos. Debido a limitaciones físicas en la construcción de sensores térmicos, es razonable asumir que la resolución de los sensores térmicos no igualará a la de otros sensores (espectro visible e infrarrojo cercano) en el corto y medio término. Desde los años 1970, variedad de algoritmos han sido desarrollados en Teledetección para mejorar la resolución de sensores de baja resolución con información de imágenes de mayor resolución. Estos algoritmos, originalmente diseñados para plataformas satélite, son llamados pansharpening. Se han realizado investigaciones previas con el objetivo de trasladar estas técnicas a imágenes térmicas. La extensión de estos estudios previos fue analizar sólo uno de los diferentes algoritmos de pansharpening existentes. En nuestro trabajo se han estudiado más de diez algoritmos de las dos principales familias de algoritmos de pansharpening para determinar sus posibilidades, funcionamiento y resultados cuando se aplican a imágenes térmicas, enfocados a aquellas obtenidas desde un dron. Nuestra metodología simula una imagen térmica de menor resolución, que una vez combinada con imágenes de espectro visible, y procesadas, pueden ser comparadas con aquellas imágenes térmicas en la resolución original, para establecer la calidad de la fusión. Esta metodología se ha aplicado en una campaña de adquisición de imágenes térmicas y de espectro visible sobre un edificio industrial cerca de Toledo (España). La calidad de los productos finales se ha calculado cuantitativamente. Investigaciones anteriores no analizaban el desempeño en parámetros numéricos medibles y comparables. Sus resultados sólo eran analizados visualmente y era imposible asegurar la calidad en procesos y análisis siguientes usando estas imágenes mejoradas. Aqui, hemos calculado índices de calidad de las imagenes térmicas mejoradas, llegando a determinar valores de calidad como los correspondientes al algoritmo de pansharpening BDSD: RMSE $=7.400$, ERGAS $=1.084$, SAM $=0.048, \mathrm{PSNR}=31.014, \mathrm{UQI}=0.995$. En conclusión, hemos validado el potencial de los algoritmos de pansharpening para mejorar la resolución de imágenes térmicas con la ayuda de imágenes de espectro visible de mayor resolución.

Palabras clave: imagen térmica, aumento de resolución, pansharpening, superresolución 


\section{Introducción}

Las cámaras térmicas, con sensores sensibles a la parte infrarroja de onda larga del espectro electromagnético (longitudes de onda desde 9 hasta 14 micrómetros), se están convirtiendo en herramientas de uso común. Pero, a diferencia de otro tipo de sensores, como las cámaras de espectro visible, la resolución de los sensores térmicos apenas ha aumentado en los últimos años. Los sensores más avanzados difícilmente sobrepasan la frontera del megapíxel. Las cámaras termográficas más empleadas están basadas en sensores no refrigerados que funcionan a temperatura ambiente denominados microbolómetros. Estos microbolómetros reciben la radiación, absorbiéndola y calentándose, variando así su resistencia eléctrica. Limitaciones técnicas en la fabricación de los microbolómetros hacen difícil su miniaturización: la relación señal/ruido es inversamente proporcional a su tamaño. Por lo que se puede afirmar que la resolución de los sensores térmicos, al menos con esta tecnología, no igualará a la de otros sensores en el corto y medio plazo.

Los distintos fabricantes de cámaras térmicas han seguido distintas estrategias con objetivo de mejorar la resolución de sus imágenes térmicas. El fabricante FLIR, con su tecnología Ultramax@, combina varias tomas ligeramente diferentes por los inevitables movimientos y vibraciones durante la captura. Este fabricante asegura doblar la resolución con esta técnica. Otro fabricante, Infratec, ha diseñado una solución tipo hardware donde una rueda giratoria a alta velocidad toma cuatro imágenes, fusionándolas en la imagen final.

Otras técnicas orientadas a la mejora de la resolución de imágenes térmicas utilizan herramientas de aprendizaje profundo (Deep Learning), donde se proporciona la imagen térmica y la correspondiente a una cámara "tradicional" de espectro visible. Pasando a través de la arquitectura de red neuronal diseñada, obtiene como producto final la imagen térmica con superresolución. Estas técnicas necesitan un entrenamiento previo con un gran conjunto de imágenes, por lo que puede no ser adecuado para todas las situaciones.

Ningún trabajo hasta la fecha ha analizado un amplio conjunto de algoritmos de pansharpening aplicados a imágenes térmicas, con el objetivo de determinar sus posibilidades. Hay que aclarar que todos los algoritmos de pansharpening existentes han sido diseñados, en origen, para imágenes de satélite. Así mismo, tampoco aparece en la literatura científica, ningún análisis cuantitativo de la calidad de los resultados de pansharpening sobre imágenes térmicas. Este trabajo ha realizado este estudio, orientado a su empleo con imágenes térmicas procedentes de plataformas UAV, aunque es extrapolable a otras situaciones.

El objetivo de este trabajo es determinar el desempeño de un gran número de algoritmos de pansharpening en la mejora de resolución de imágenes térmicas al fusionarlas con imágenes de espectro visible. Para ello, hemos diseñado una metodología para poder conocer la calidad de los productos de estos algoritmos, mediante índices de calidad.

\section{Métodos y Materiales}

Las imágenes multiespectrales son aquellas compuestas por distintas bandas que representan partes diferentes del espectro electromagnético. Las bandas típicas de estas imágenes corresponden a colores dentro del espectro visible (rojo, verde y azul), junto a otras bandas como aquellas procedentes del infrarrojo cercano (NIR), el infrarrojo de onda corta (SWIR) o partes del espectro ultravioleta.

En resumen, podemos definir una imagen multiespectral como un conjunto de imágenes (normalmente entre 3 y 15) correspondientes a la misma toma con diferentes partes del espectro electromagnético.

Las imágenes térmicas normalmente son expresadas con distintas mascaras de color, definiendo así una imagen en falso color (Fig. 1a). Esto ayuda a un correcto análisis y hace más fácil al usuario la interpretación de la información aportada por la imagen térmica. La carta de color más común expresa temperaturas menores con colores fríos (azules y violetas) y temperaturas más altas con colores cálidos (amarillos, naranjas y rojos). Aunque esto es una representación artificial de los valores brutos de la imagen térmica, nos ayudará a componer nuestra imagen pseudo-multiespectral (PS-MS).

La imagen pseudo-multiespectral (PS-MS) es aquella compuesta por las tres bandas RGB de la imagen térmica en falso color (Fig. 1a) junto a la imagen correspondiente a los valores térmicos brutos en escala de grises (Fig. 1b).

Para comprobar el desempeño de los distintos algoritmos, hemos simulado una imagen pseudomultiespectral en baja resolución (PS-MS LR). Aplicando un algoritmo piramidal gaussiano con ratio 4 y $\sigma=4 / 3$, logramos esta imagen pseudo-multiespectral en baja resolución. En ella se aplicaron los algoritmos de pansharpening para la mejora de su resolución y así analizamos su calidad cuantitativamente con índices de calidad.

Otro de los elementos comunes a todos los algoritmos de pansharpening, corresponde a la necesaria imagen pancromática (PAN). Esta imagen debe proceder de un sensor de mayor resolución. Usualmente, las cámaras térmicas, junto al sensor térmico, montan un sensor de espectro visible RGB, que ayuda al correcto encuadre de la escena, pues las imágenes térmicas en ocasiones no presentan contraste. Este sensor de espectro visible tiene mucha mayor resolución por lo que nos aportó la imagen pancromática PAN necesaria, expresando la imagen RGB en escala de grises.

Entonces pudimos aplicar todos los algoritmos de pansharpening a estudio sobre la imagen PS-MS_LR, que al fusionarse con la imagen PAN, nos dio como resultado la imagen pseudo multiespectral mejorada (PSMS_HR*). Esta imagen producto está compuesta, como la inicial, por tres bandas RGB correspondientes al falso color y una cuarta banda en escala de grises. Para posteriores análisis de calidad, se separó este producto final en dos imágenes a su vez: la correspondiente en falso color y la térmica "bruta" en escala de grises. 


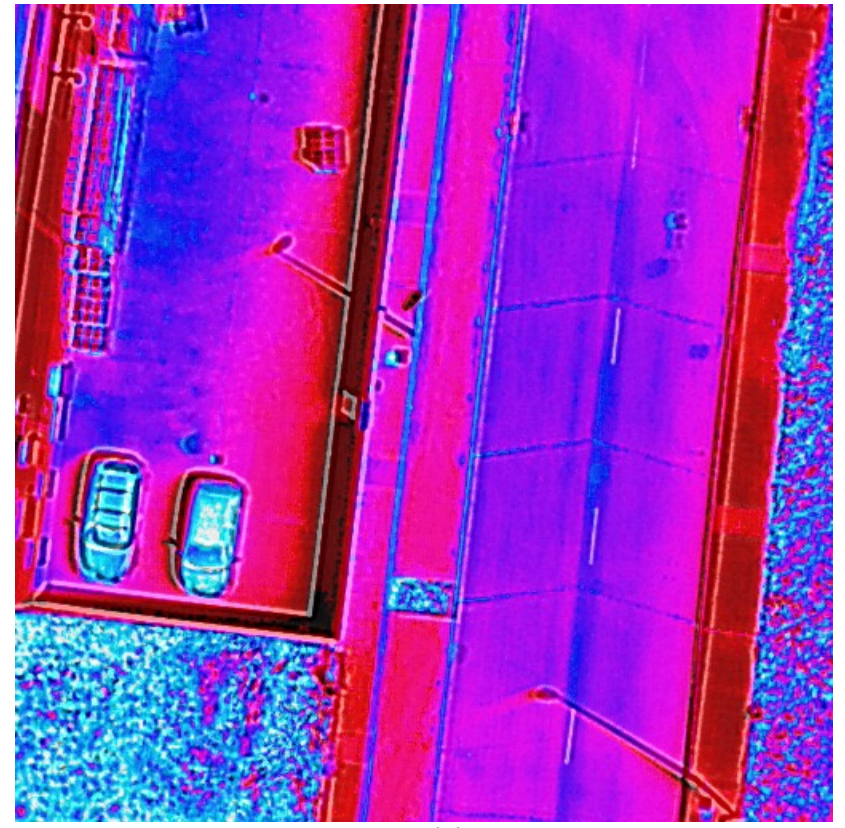

(a)

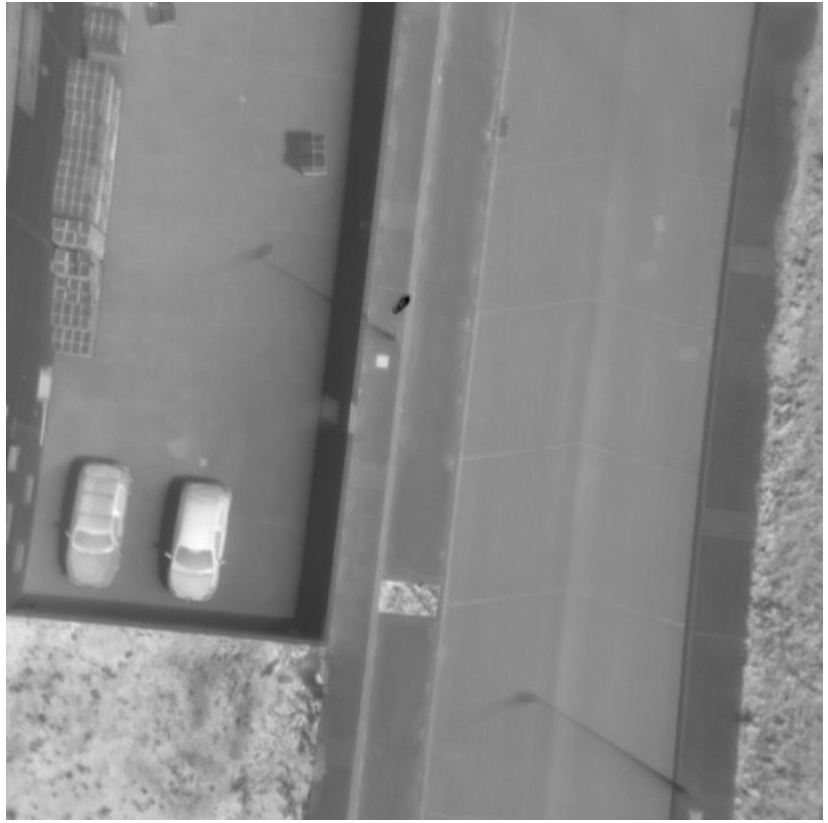

(b)

Figura 1: Imagen térmica a resolución original: a) en falso color; b) en escala de grises

\subsection{Algoritmos de pansharpening}

Los algoritmos de pansharpening pertenecen, dentro del campo de la teledetección, a la rama de fusión de imágenes. Su objetivo es mejorar imágenes de baja resolución usando imágenes procedentes de otros sensores de mayor resolución. Hay que aclarar que ambas imágenes deben mostrar el mismo objeto con el mismo campo de visión.

Existen dos familias de algoritmos de pansharpening bien definidas en la literatura científica. Se diferencian principalmente por su aproximación al problema: espacial o espectral.

Los algoritmos de SUSTITUCION DE COMPONENTES (CS) se basan en la transformación del espacio de color de la imagen multiespectral, desasociando la información espacial y la espectral. La información espacial es posteriormente sustituida por aquella procedente de la imagen de mayor resolución. Aplicando la transformación del espacio de color a la inversa, obtenemos la imagen multiespectral con resolución mejorada. Los algoritmos de sustitución de componentes son globales, pues actúan de manera uniforme por toda la extensión de la imagen (Chang and Bai 2018).

Por otro lado, los métodos de Análisis Multirresolución (MRA) usan filtros lineales invariantes en el espacio para extraer los detalles espaciales de la imagen de alta resolución y añadírselos a la imagen multiespectral (Chen 2012).

En este trabajo se han analizado los siguientes algoritmos de pansharpening aplicados a imágenes térmicas:

- $\quad$ IHS: Fusión rápida de imágenes por tono, saturación e intensidad (Tu et al. 2001) (Fig. 2).

- PCA: Análisis de Componentes Principales (Chavez et al. 1991) (Fig. 3).
- BDSD: Detalles espaciales dependientes de banda con estimación de parámetros locales (Garzelli et al. 2008) (Flg. 4).

- GS: Transformación Gram Schmidt (modo 1) (Laben and Brower 2000).

- PRACS: Reemplazo parcial adaptativo con sustitución de componentes (Choi et al. 2011).

- HPF: Filtrado de paso alto $5 \times 5$ con fusión 1:4 (Chavez et al. 1991).

- SFIM: Filtro de suavizado basado en modulación de intensidad (Liu 2000).

- INDUSION: Transformación de ondícula decimada usando modelo de inyección aditiva (Khan et al. 2008).

- $\quad$ MTF-GLP: Función de transferencia de modulación con piramidal laplaciana generalizada y modelo de inyección unitario (Aiazzi et al. 2002).

- MTF-GLP-HPM: Similar a MTF-GLP, con modelo de inyección multiplicativa (Aiazzi et al. 2006).

- MTF-GLP-HPM-PP: Similar a MTF-GLP-HPM, con post proceso (Aiazzi et al. 2006).

- MTF-GLP-ECB: Similar a MTF-GLP, con algoritmo de modelo de mejora basado en contexto (Aiazzi et al. 2006).

Los algoritmos IHS, PCA, GS, BDSD y PRACS pertenecen a la categoría de algoritmos de pansharpening por sustitución de componentes (CS), mientras que HPF, SFIM, INDUSION y las diferentes versiones de MTF son del grupo de análisis multirresolución (MRA). Todos estos algoritmos han sido computados usando la librería MATLAB distribuida por Vivone et al. (2019). 


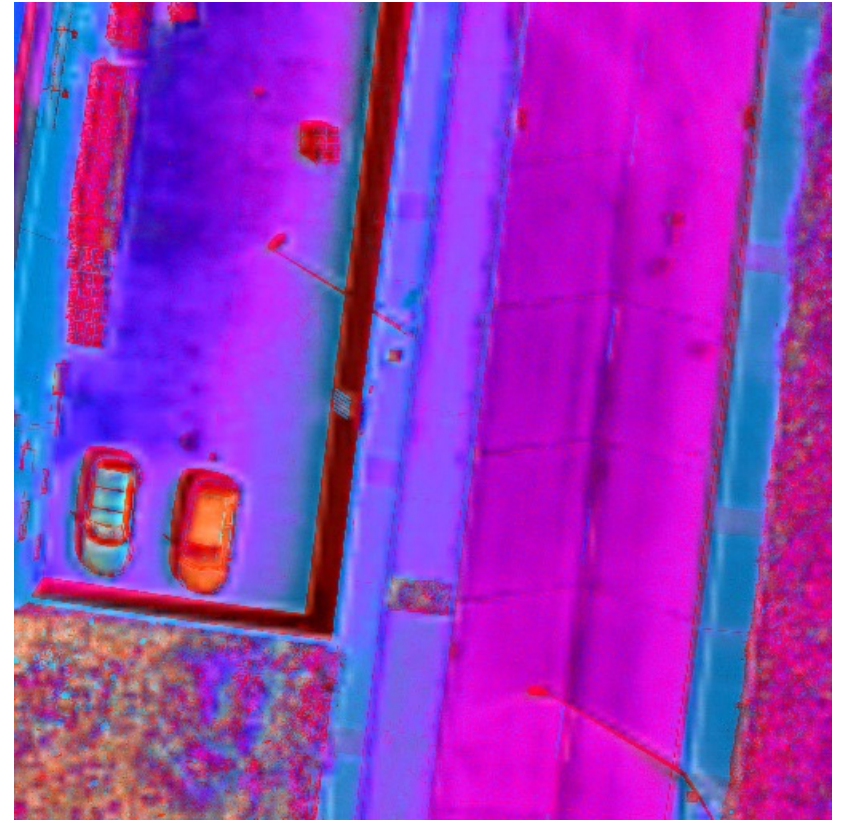

(a)

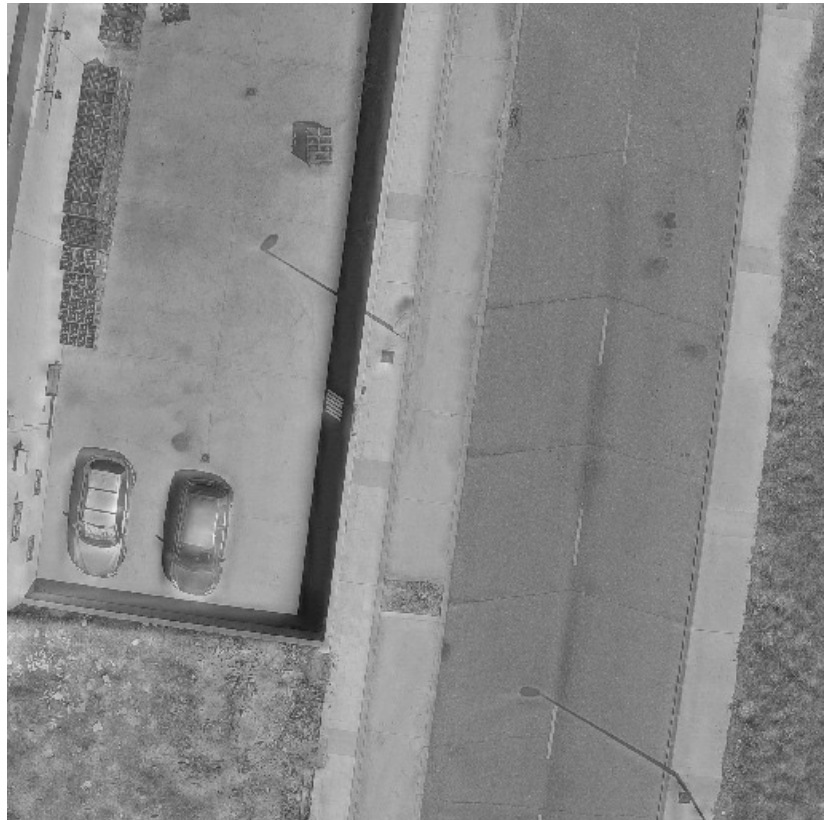

(b)

Figura 2: Imagen térmica de resolución mejorada por algoritmo PCA: a) en falso color; b) en escala de grises

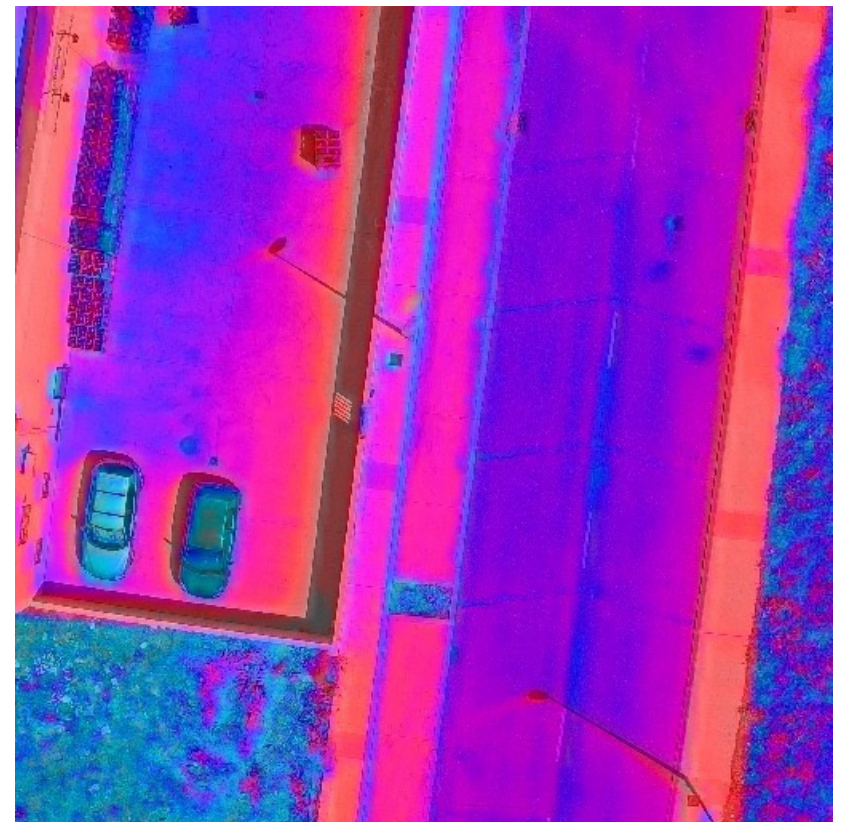

(a)

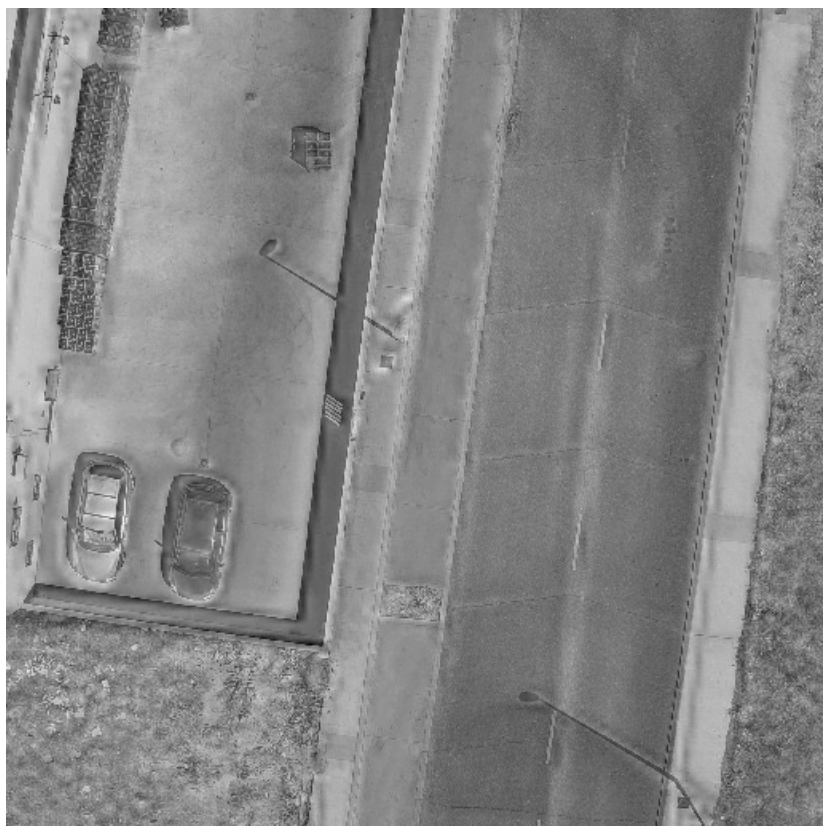

(b)

Figura 3: Imagen térmica de resolución mejorada por algoritmo IHS: a) en falso color; b) en escala de grises

Una vez establecido la extensión de nuestro estudio, definimos las características que los productos finales para medirlas en la valoración cuantitativa.

\subsection{Protocolo de Wald}

Antes de su uso posterior, las imágenes resultantes de la mejora de resolución de las imágenes térmicas por estos algoritmos de pansharpening deben ser evaluadas mediante índices de calidad cuantitativos. Una evaluación visual es del todo insuficiente para comprobar su idoneidad.

Entre la comunidad investigadora está aceptado el denominado protocolo de Wald (Wald et al. 1997) como el que establece las propiedades esenciales que deben presentar los productos de fusión de imágenes siempre que sea posible. Este protocolo se define en tres teoremas:

- Teorema 1. Consistencia: Toda imagen fusionada, una vez degradada a su resolución original, debe ser lo más idéntica posible a la imagen original.

- Teorema 2. Síntesis: Toda imagen fusionada con otra de mayor resolución debe ser lo más idéntica posible a la imagen ideal que correspondería al sensor correspondiente, si existiera, a la imagen de alta resolución

- Teorema 3. El vector de imágenes multiespectrales fusionadas con una de mayor resolución debe ser lo 
más idéntica posible al vector de imágenes multiespectrales ideal que correspondería al sensor, si existiera, con la resolución espacial de la imagen de alta resolución.

Como gracias a la metodología diseñada dispusimos de la imagen con la resolución original se cumple con los teoremas 2 y 3 del protocolo de Wald.

La calidad de las imágenes resultado de los algoritmos de pansharpening debe ser medida. Una inspección visual se hace necesaria pero no suficiente. Varios índices de calidad de fusión de imágenes han sido propuestos para asegurar la calidad de esta fusión.

\section{3. Índices de Calidad}

Los índices de calidad de fusión de imágenes miden la distorsión espacial y espectral basados en distintos estadísticos con variaciones entre ellos. Mientras unos se focalizan en la reconstrucción espacial otros son diseñados para evaluar la variación espectral.

En este estudio se han seleccionado y calculado los siguientes índices:

- RMSE (Root Mean Squared Error): Raíz del error medio cuadrático entre las dos imágenes. Expresa ambas distorsiones (espacial y espectral). Su valor optimo es cero.

- $\quad$ ERGAS (Erreur Relative Globale Adimensionnelle de Synthèse): Propuesto por Ranchin and Wald (2000), es un estadístico global que expresa la calidad de la imagen final. Mide la transición entre la información espacial y espectral. Su valor optimo es 0 .

- SAM: Ángulo de mapa espectral. Mide la distorsión espectral por el ángulo formado por los dos vectores del espectro de ambas imágenes. Su valor optimo es 0 .

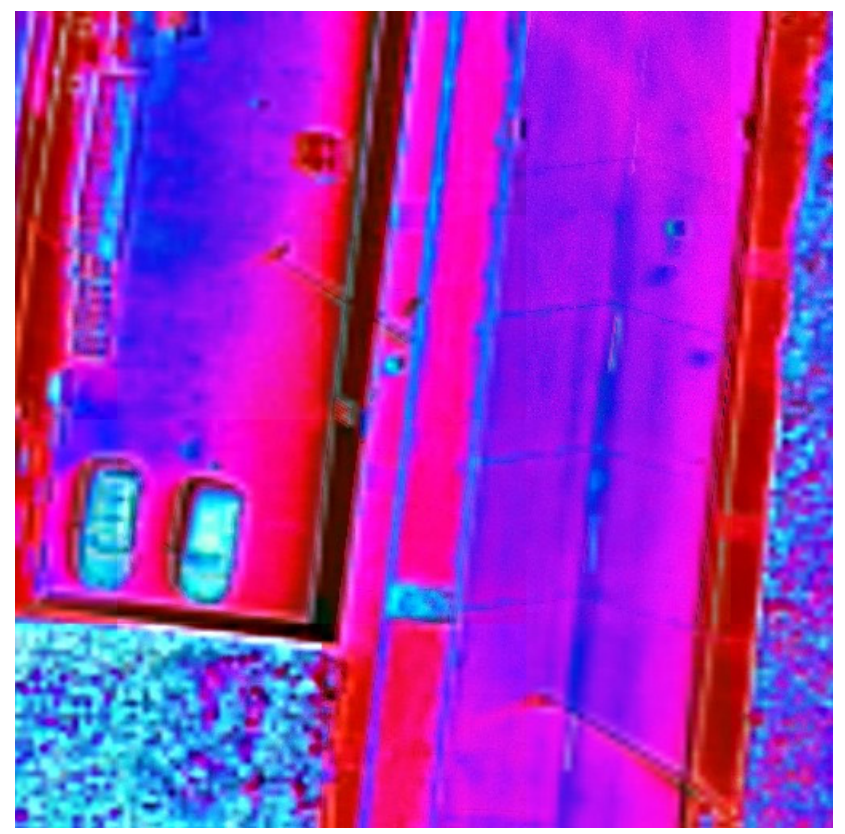

(a)
- PSNR: Proporción máxima de señal a ruido. Describe la reconstrucción espacial de las imágenes finales. Si las dos imágenes fueran idénticas, la PSNR tendería a infinito.

- UQI: Índice de calidad universal, propuesto por Wang and Bovik (2002), estima la distorsión combinando tres factores: pérdida de correlación, distorsión de la luminancia y distorsión del contraste. Los valores de UQI se mueven dentro del intervalo $[-1,1]$ siendo 1 el valor óptimo.

Todos estos índices se calcularon de las imágenes procesadas de manera diferenciada. La imagen pseudomultiespectral mejorada (PS-MS_HR*) se separó en dos: la imagen con las tres bandas de falso color y la última banda en escala de grises, que se compararon con sus originales correspondientes. Esto nos permitió distinguir la calidad de la transformación de modo independiente de la máscara de falso color elegida.

\subsection{Datos}

Para testar el resultado de los algoritmos de pansharpening aplicados a imágenes térmicas, se tomó un conjunto de imágenes con las características necesarias. Estas imágenes proceden de una misión tomada por un vehículo aéreo pilotado remotamente (UAV) sobre un edificio industrial localizado en la localidad de Illescas (Toledo - España) el día 13 de agosto de 2019.

El vehículo aéreo estaba equipado con dos sensores. Un sensor de espectro visible RGB 4K CMOS con una resolución de imagen de 3840 x 2160 pixeles, junto a un sensor infrarrojo térmico radiométrico por microbolómetro VOx, cuyas imágenes tienen $640 \times 512$ pixeles.

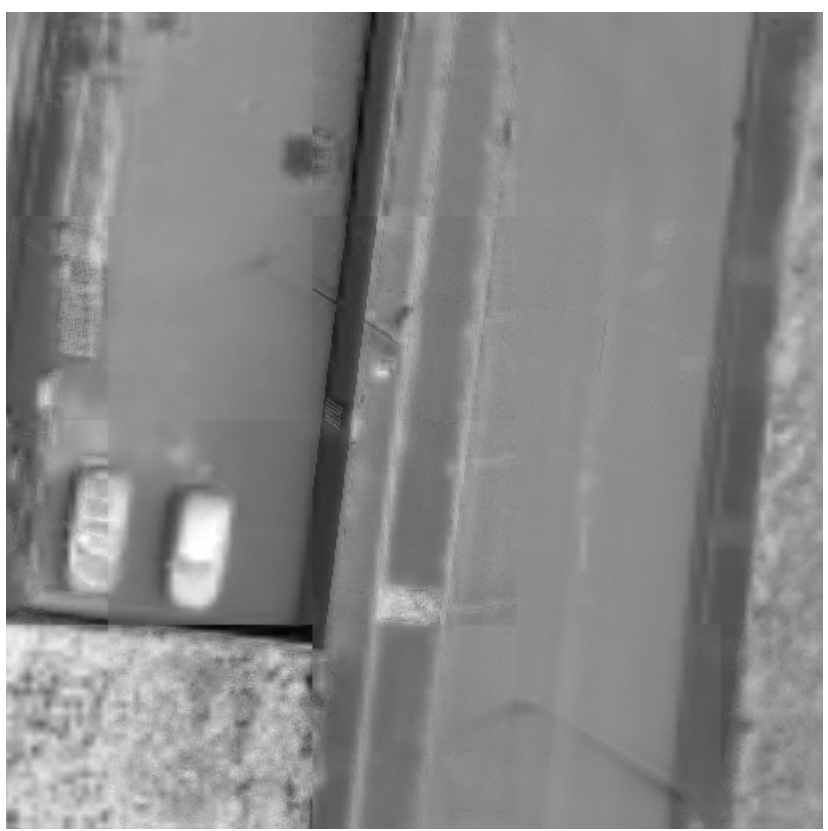

(b)

Figura 4: Imagen térmica de resolución mejorada por algoritmo BDSD: a) en falso color; b) en escala de grises. 
Como la plataforma aérea equipaba ambos sensores con lentes y resoluciones diferentes, es necesario un trabajo previo de alineación. El alineamiento de imágenes consiste en transformar una imagen de forma que entidades de ambas imágenes queden perfectamente alineadas en el mismo campo de visión. Para ello, identificando entidades comunes en ambas imágenes, se calculan los parámetros de la transformación afín que transformará la imagen visual, por su mayor resolución, en el mismo campo que la imagen térmica.

El alineamiento no afectó al desempeño de los algoritmos de pansharpening pues así, ambas imágenes (térmica y visible) representaban el mismo objeto.

\section{Resultados}

Como se ha indicado en el apartado de metodología, las imágenes térmicas procedentes del vuelo UAV se han procesado siguiendo los pasos necesarios para obtener la imagen pseudo multiespectral en la resolución original (PS-MS HR) y la imagen pseudo multiespectral en baja resolución (PS-MS_LR). Por otro lado, la imagen de la cámara de espectro visible RGB, después del alineamiento necesario, se expresó en escala de grises dando lugar a la imagen pancromática (PAN).

Aplicando todos los algoritmos de pansharpening seleccionados a la imagen en baja resolución (PSMS_LR) junto a la imagen pancromática (PAN), el producto final se denomina imagen pseudomultiespectral mejorada (PS-MS_HR*) correspondiente a cada uno de ellos.

Los índices de calidad se han calculado, como se ha comentado, de forma diferenciada: por un lado, la imagen en falso color y por otro la imagen térmica en escala de grises. Los índices se han calculado sobre la imagen en resolución original (PS-MS_HR) y la imagen de resolución mejorada (PS-MS_HR*). En las Tablas 1 y 2 se muestran los valores obtenidos para cada uno de los distintos algoritmos.

\section{Discusión}

Una vez analizados los resultados obtenidos, podemos afirmar:

- Los resultados respecto a las imágenes en falso color y la correspondiente en escala de grises son cuantitativamente diferentes. Las imágenes térmicas en escala de grises se comportan mucho mejor que aquellas en falso color, como se muestra en las Tablas 1 y 2. El índice RMSE de las imágenes en escala de gris es similar o incluso más bajo que en otros trabajos de investigación consultados (Mandanici et al. 2019). Por ello, las imágenes en escala de grises deben ser las utilizadas en procesos posteriores, incluso en el caso de aplicar la misma u otra mascara de falso color.

- Salvo algunos valores específicos, las dos familias de algoritmos de pansharpening obtienen valores similares en los índices de calidad. Diferencias menores en la manera en que los distintos algoritmos procesan los datos producen mejores resultados. En la familia de algoritmos de sustitución de componentes, el algoritmo BDSD obtiene mejores resultados que el resto.
- En general, los algoritmos de análisis multirresolución (MRA) funcionan mejor que los métodos de sustitución de componentes (CS). Entre todos ellos, el algoritmo BDSD es el que mejores resultados obtiene $(\mathrm{RMSE}=7.400, \mathrm{ERGAS}=1.084$, SAM $=0.048$, PSNR $=31.014$, UQI $=0.995)$. Haselwimmer (2013) sugiere que el mejor algoritmo para mejorar imágenes térmicas es IHS, pero aquí demostramos que no es la mejor opción.

Tabla 1: Índices de calidad (valores medios) de imagenes mejoradas en falso color.

\begin{tabular}{c|c|c|c|c|c} 
Algoritmo & $R M S E$ & $E R G A S$ & $S A M$ & $P S N R$ & UQI \\
\hline PCA & 66.084 & 40.524 & 0.323 & 11.883 & 0.854 \\
IHS & 46.524 & 27.716 & 0.224 & 14.798 & 0.891 \\
BDSD & 26.167 & 17.235 & 0.125 & 19.789 & 0.925 \\
GS & 88.872 & 29.372 & 0.438 & 9.241 & 0.798 \\
PRACS & 42.932 & 17.977 & 0.207 & 15.537 & 0.919 \\
HPF & 38.962 & 23.463 & 0.187 & 16.350 & 0.947 \\
SFIM & 44.139 & 29.593 & 0.212 & 15.284 & 0.951 \\
$\begin{array}{c}\text { INDUSION } \\
\text { MTF-GLP }\end{array}$ & 40.867 & 24.698 & 0.197 & 15.951 & 0.917 \\
$\begin{array}{c}\text { MTF-GLP- } \\
\text { HPM }\end{array}$ & 44.432 & 29.585 & 0.190 & 16.243 & 0.945 \\
$\begin{array}{c}\text { MTF-GLP- } \\
\text { HPM_PP }\end{array}$ & 42.047 & 39.971 & 0.202 & 15.675 & 0.950 \\
$\begin{array}{c}\text { MTF-GLP- } \\
\text { ECB }\end{array}$ & 43.876 & 33.677 & 0.211 & 15.314 & 0.931 \\
$\begin{array}{c}\text { MTF-GLP- } \\
\text { CBD }\end{array}$ & 30.612 & 18.023 & 0.147 & 18.503 & 0.959 \\
& & & & &
\end{tabular}

Tabla 2: Índices de calidad (valores medios) de imagenes mejoradas "brutas", en escala de gris

\begin{tabular}{c|c|c|c|c|c} 
Algoritmo & $R M S E$ & $E R G A S$ & $S A M$ & $P S N R$ & UQI \\
\hline PCA & 31.774 & 5.209 & 0.208 & 18.353 & 0.954 \\
IHS & 39.167 & 5.837 & 0.250 & 16.322 & 0.940 \\
BDSD & 7.400 & 1.084 & 0.048 & 31.014 & 0.995 \\
GS & 32.743 & 4.974 & 0.211 & 17.915 & 0.954 \\
PRACS & 25.352 & 2.885 & 0.159 & 20.934 & 0.972 \\
HPF & 15.108 & 2.274 & 0.096 & 24.693 & 0.994 \\
SFIM & 16.610 & 2.436 & 0.106 & 23.847 & 0.994 \\
INDUSION & 16.848 & 2.565 & 0.106 & 23.754 & 0.991 \\
MTF-GLP & 15.441 & 2.315 & 0.098 & 24.496 & 0.993 \\
MTF-GLP- & 16.892 & 2.471 & 0.107 & 23.693 & 0.994 \\
HPM & & & & & \\
$\begin{array}{c}\text { MTF-GLP- } \\
\text { HPM_PP }\end{array}$ & 17.210 & 2.540 & 0.109 & 23.519 & 0.993 \\
$\begin{array}{c}\text { MTF-GLP- } \\
\text { ECB }\end{array}$ & 26.334 & 3.640 & 0.168 & 19.917 & 0.982 \\
$\begin{array}{c}\text { MTF-GLP- } \\
\text { CBD }\end{array}$ & 8.567 & 1.283 & 0.054 & 30.125 & 0.997 \\
& & & & &
\end{tabular}


- $\quad$ Desde el punto de vista radiométrico, no hay una opción claramente optima respecto a las otras. Los indices ERGAS y SAM tienen valores similares entre los distintos métodos, aunque los algoritmos MRA funcionan algo mejor. Esto concuerda con el comportamiento general descrito en la literatura (Aiazzi et al. 2009).

- La reconstrucción espacial es mejor en los algoritmos de análisis multirresolución. El índice PSNR es mayor, informando de una mayor calidad geométrica de los detalles espaciales. De nuevo, el algoritmo BDSD es el mejor en términos de reconstrucción espacial.

- Nuestro trabajo permite el uso de sensores térmicos con menor resolución a la de otros usados simultáneamente en el mismo proyecto, debido a que estos algoritmos mejoran y homogeneizan la resolución. Una limitación es que es dependiente de la razón de resolución entre los distintos sensores. Una razón entre las resoluciones mayor a 4 puede provocar artefactos inesperados y el fallo de los procesos (Dumitrescu and Boiangiu 2019)

- Aunque los resultados pueden ser dependientes de la máscara de falso color elegida para expresar la información térmica en la imagen pseudomultiespectral, la validación de los algoritmos de pansharpening en las imágenes térmicas aquí demostrada subraya el interés en futuros desarrollos e investigaciones para ajustar los parámetros de los algoritmos para adaptarlos específicamente a las imágenes térmicas.

\section{Conclusiones}

El uso de ciertos algoritmos de pansharpening aplicados a imágenes térmicas había sido estudiado en investigaciones anteriores. Nuestro trabajo contiene una completa revisión de un amplio número de algoritmos, y provee de un estudio en profundidad de su funcionamiento y un análisis cuantitativo, no realizado hasta la fecha.

Nos hemos enfocado en imágenes procedentes de plataformas aéreas UAV como aplicación principal, pues su uso es cada vez más común en la inspección de instalaciones y edificios, por su versatilidad.

La disponibilidad de una estimación precisa de la calidad de los productos de algoritmos de pansharpening sobre imágenes térmicas hará en el futuro más fácil el desarrollo de sistemas de teledetección robustos y fiables.

\section{Agradecimientos}

Queremos agradecer a Vivone et al. (2015) por hacer disponibles para la comunidad investigadora las herramientas MATLAB de algoritmos de pansharpening, y a los componentes del grupo de investigación Geovisualización, Espacios Singulares y Patrimonio (GESyP) de la Universidad Politécnica de Madrid (UPM) por su trabajo y apoyo.

El primer autor, Javier Raimundo, quiere agradecer al Consejo General de la Arquitectura Técnica de España (CGATE) por su apoyo.

\section{References}

AIAZZI, B., ALPARONE, L., BARONTI, S., and GARZELLI, A., 2002. Context-driven fusion of high spatial and spectral resolution images based on oversampled multiresolution analysis. IEEE Transactions on geoscience and remote sensing, 40(10), pp. 2300-2312. DOI: 10.1109/TGRS.2002.803623

AIAZZI, B., ALPARONE, L., BARONTI, S., GARZELLI, A., and SELVA, M., 2006. MTF-tailored multiscale fusion of highresolution MS and Pan imagery. Photogrammetric Engineering \& Remote Sensing, 72(5), pp. 591-596. DOI: 10.14358/PERS.72.5.591

AIAZZI, B., BARONTI, S., LOTTI, F., and SELVA, M., 2009. A comparison between global and context-adaptive pansharpening of multispectral images. IEEE Geoscience and Remote Sensing Letters, 6(2), pp. 302-306. DOI: 10.1109/LGRS.2008.2012003

CHANG, N. B., and BAI, K., 2018. Multisensor data fusion and machine learning for environmental remote sensing. CRC Press. DOI: 10.1201/9781315154602

CHAVEZ, P., SIDES, S. C., and ANDERSON, J. A., 1991. Comparison of three different methods to merge multiresolution and multispectral data- Landsat TM and SPOT panchromatic. Photogrammetric Engineering and remote sensing, 57(3), pp. 295-303. DOI: 10.1306/44b4c288-170a-11d7-8645000102c1865d

CHEN, C. H., 2012. Land Cover Estimation with Satellite Image Using Neural Network. In Signal and Image Processing for Remote Sensing (pp. 546-553). CRC Press. DOI: 10.1201/b11656-30

CHOI, J., YU, K., and KIM, Y., 2010. A new adaptive component-substitution-based satellite image fusion by using partial replacement. IEEE Transactions on Geoscience and Remote Sensing, 49(1), pp. 295-309. DOI: 10.1109/TGRS.2010.2051674

DUMITRESCU, D, and BOIANGIU, C.A., 2019. A Study of Image Upsampling and Downsampling Filters. Computers, 8(2), p. 30. DOI: $10.3390 /$ computers 8020030 .

GARZELLI, A., NENCINI, F. and CAPOBIANCO, L., 2008. Optimal MMSE pan sharpening of very high resolution multispectral images. IEEE Transactions on Geoscience and Remote Sensing, 46(1), pp. 228-236. DOI: 10.1109/TGRS.2007.907604 
HASELWIMMER, C., 2013. Thermal remote sensing Sensors, Methods, Applications. In Kuenzer, C. and Dech, S. (eds) Remote Sensing and Digital Image Processing. Dordrecht: Springer Netherlands, pp. 287-313. DOI: 10.1007/978-94007-6639-6

KHAN, M. M., CHANUSSOT, J., CONDAT, L., and MONTANVERT, A., 2008. Indusion: Fusion of multispectral and panchromatic images using the induction scaling technique. IEEE Geoscience and Remote Sensing Letters, 5(1), pp. 98-102. DOI: 10.1109/LGRS.2007.909934

MANDANICI, E., TAVASCI, L., CORSINI, F., and GANDOLFI, S., 2019. A multi-image super-resolution algorithm applied to thermal imagery. Applied Geomatics, 11(3), pp. 215-228. DOI: 10.1007/s12518-019-00253-y

LABEN, C. and BROWER, B., 2000. Process for enhancing the spatial resolution of multispectral imagery using pansharpening. United States Patent 6(11), p. 875.

LIU, J. G., 2000. Smoothing Filter-based Intensity Modulation: A spectral preserve image fusion technique for improving spatial details. International Journal of Remote Sensing, 21(18), pp. 3461-3472. DOI: 10.1080/014311600750037499

RANCHIN, T. and WALD, L., 2000. Fusion of high spatial and spectral resolution images: The ARSIS concept and its implementation. Photogrammetric Engineering and Remote Sensing, 66(1), pp. 49-61.

TU, T. M., SU, S. C., SHYU, H. C., and HUANG, P. S., 2001. A new look at IHS-like image fusion methods. Information fusion, 2(3), pp. 177-186. DOI: 10.1016/S1566-2535(01)00036-7

VIVONE, G., ALPARONE, L., CHANUSSOT, J., DALLA MURA, M., GARZELLI, A., LICCIARDI, G. A., and WALD, L., 2014. A critical comparison among pansharpening algorithms. IEEE Transactions on Geoscience and Remote Sensing, 53(5), pp. 2565-2586. DOI: 10.1109/TGRS.2014.2361734

WALD, L., RANCHIN, T. and MANGOLINI, M., 1997. Fusion of satellite images of different spatial resolutions: Assessing the quality of resulting images. Photogrammetric Engineering and Remote Sensing, 63(6), pp. 691-699.

WANG, Z. and BOVIK, A. C., 2002. A universal image quality index. IEEE Signal Processing Letters, 9(3), pp. 81-84. DOI: $10.1109 / 97.995823$ 